\title{
ESTUDIO PSICOMÉTRICO DE LA VERSIÓN ESPAÑOLA DEL CUESTIONARIO DE AGRESIÓN DE BUSS Y PERRY
}

\author{
PSYCHOMETRIC STUDY OF SPANISH VERSION OF AGGRESSION \\ QUESTIONNAIRE BUSS AND PERRY
}

María Matalinares C. ${ }^{1}$, Juan Yaringaño L., Joel Uceda E., Erika Fernández A., Yasmin Huari T., Alonso Campos G., Nayda Villavicencio C. Universidad Nacional Mayor de San Marcos, Lima, Perú

(RECIBIDO 15/3/2012; ACEPtAdo 16/6/2012)

\begin{abstract}
RESUMEN
El objetivo básico de la presente investigación fue realizar la adaptación psicométrica de la versión española del Cuestionario de Agresión (Buss y Perry, 1992; Andreu, Peña y Graña, 2002). Se trabajó con 3,632 sujetos, de 10 a 19 años, de ambos sexos, de $1^{\circ}$ a $5^{\circ}$ de secundaria, procedentesde distintas instituciones educativas de la costa, sierra y selva del Perú. El estudio analiza la estructura del cuestionario mediante el análisis factorial exploratorio evidenciando el modelo planteado por Bussy determina la consistencia interna aceptable. Los resultados obtenidos demuestran un ajuste aceptable al modelo de cuatro factores, una consistencia interna adecuada, baremos percentilares y normas interpretativas.
\end{abstract}

Palabras clave: Cuestionario de Agresividad, adaptación, confiabilidad, validez.

\begin{abstract}
The basic objective of this research was to conduct psychometric adaptation of the Spanish version of the Aggression Questionnaire (Buss and Perry, 1992; Andreu, Peña and Grana, 2002). It was worked with 3,632 from 10 to 16 years, of both sexes, of $1^{\circ}$ to $5^{\circ}$ degree of secondary education, fromdifferent educational institutions of the coast, highlands and jungle of Peru. This study analyzes the structure of the questionnaire through exploratory factor analysis evidencing the model proposed by Buss and determines acceptable internal consistency. The results obtained show an acceptable fit to the four-factor model, adequate internal consistency, scales percentile and interpretative rules.
\end{abstract}

Keywords: aggression questionnaire, adaptation, reliability, validity. 


\section{INTRODUCCIÓN}

El presente estudio surge a partir de los estudios de Arnold Buss sobre la agresividad, teniendo en cuenta que la agresión o conducta agresiva es una acción externa, abierta, objetiva y observable, que a lo largo de los años se ha ido definiendo con distintas formulaciones. Buss en 1961 señaló: "La distinción entre agresión de enojo y la agresión instrumental. Cuando es agresión de enojo, son recompensados por el dolor o el malestar de las víctimas de nuestra agresión. Cuando no se está enojado, nuestra agresión es recompensada por cualquiera de los muchos reforzadores que se producen en la vida cotidiana (dinero, estado, y así sucesivamente); de este modo los agresivos logran las mismas recompensas que los no agresivos, de ahí surge el termino de agresión instrumental. El Cuestionario de Agresión surge a partir de los trabajos de Buss y Perry (1992) que determinaron la construcción del denominado Aggression Questionnaire (AQ), A este cuestionario se realizó la adaptación psicométrica en la versión española, por Andreu, Peña y Graña (2002).

Buss considera la conducta agresiva como una "respuesta que proporciona estímulos dañinos a otro organismo" (Buss, 1961). Como se puede observar en la agresión el efecto nocivo, es importante pero no se debe considerar como único factor, en otros estudios se busca conocer la intencionalidad y la participación de juicios o prácticas sociales (Bandura, 1976). Así, algunos actos agresivos pueden aprobarse como legítimos en determinadas situaciones, pero condenarse en otras distintas. De acuerdo con la teoría del aprendizaje social de Bandura (1977), estas actitudes pueden fomentar o bloquear la manifestación de conductas agresivas dentro de determinados contextos sociales. La agresión quedaría conceptualizada como aquella actividad a través de la cual una persona busca infringir daño o dolor físico sobre otra que está motivada para evitarlo. El estado agresivo se configura como una combinación de cogniciones, emociones y tendencias comportamentales desencadenadas por estímulos capaces de evocar una respuesta agresiva, aunque no sean condición necesaria para ello ya que esta puede verse desencadenada por otra serie de factores. Esta dimensión subjetiva de la agresión se ha ido caracterizando conceptualmente a través de términos tales como: agresividad, ira y hostilidad.

Estructura del cuestionario de agresión de Buss y Perry. La prueba consta de 4 dimensiones y la distribución de ítems por dimensión es la siguiente: agresión física (9), agresividad verbal (5), hostilidad (7) e ira (8).

Agresividad. Es una disposición relativamente persistente a ser agresivo en diversas situaciones. Por tanto, hace referencia a una variable interviniente que indica la actitud o inclinación que siente una persona o un colectivo humano a realizar un acto agresivo. En este sentido, puede también hablarse de potencial agresivo. La agresividad suele ser concebida como una respuesta adaptativa que forma parte de las estrategias de afrontamiento de los seres humanos a las amenazas externas. Esta se presenta como agresividad física y agresividad verbal.

Hostilidad. Se refiere a la evaluación negativa acerca de las personas y las cosas (Buss, 1961), a menudo acompañada de un claro deseo de hacerles daño o 
agredirlos (Kaufmann, 1970). Esta actitud negativa hacia una o más personas se refleja en un juicio desfavorable de ella o ellas (Berkowitz, 1996). Tal y como este autor afirma, se expresa hostilidad cuando decimos que alguien nos disgusta, especialmente si deseamos el mal para esta persona. Un individuo hostil es alguien que normalmente hace evaluaciones negativas de y hacia los demás, mostrando desprecio o disgusto global por muchas personas (Spielberger, Jacobs, Rusell y Crane, 1983).

La hostilidad implica una actitud de resentimiento que incluye respuestas tanto verbales como motoras. Plutchik (1980) la consideró como una actitud que mezcla la ira y disgusto, y se ve acompañada de sentimientos tales como indignación, desprecio y resentimiento hacia los demás. Precisamente, estos sentimientos (resentimiento, indignación y animosidad) configuran la hostilidad como una actitud de la naturaleza humana, en general, que en ocasiones puede llegar incluso al rencor y a la violencia. La hostilidad conlleva creencias negativas acerca de otras personas, así como la atribución general de que el comportamiento de los demás es agresivo o amenazador. La "atribución hostil" hace referencia precisamente a la percepción de otras personas como amenazantes y agresivas (Fernández-Abascal, 1998).

Ira. Se refiere al conjunto de sentimientos que siguen a la percepción de haber sido dañado. No persigue una meta concreta, como en el caso de la agresión, sino que hace referencia principalmente a un conjunto de sentimientos que surgen de reacciones psicológicas internas y de las expresiones emocionales involuntarias producidas por la aparición de un acontecimiento desagradable (Berkowitz, 1996). La ira implica sentimientos de enojo o enfado de intensidad variable (Spielberger, Jacobs, Rusell, y Crane, 1983). La ira es una reacción de irritación, furia o cólera que puede verse elicitada por la indignación y el enojo al sentir vulnerados nuestros derechos (Fernández-Abascal,1998). Izard (1977) la conceptualizó como una emoción básica que se expresa cuando un organismo se ve obstaculizado o impedido en la consecución de una meta o en la satisfacción de una necesidad. Diamond (1982), por otra parte, la describió como un estado de arousal o activación general del organismo con componentes expresivos, subjetivos, viscerales y somáticos.

Existen complejas relaciones entre ira, hostilidad yagresión. La ira puede ser considerada el concepto más simple de los tres. Por el contrario la hostilidad implica una actitud que usualmente va acompañada de sentimientos de enfado o ira y que predispone hacia la emisión de conductas agresivas dirigidas principalmente a la destrucción de objetos, al insulto o a la producción de algún daño. Si la ira y la hostilidad se refieren a sentimientos y actitudes, la agresión implica un paso más allá,puesto que conlleva la aparición de comportamientos destructivos, lesivos o punitivos dirigidos a otras personas u objetos (Miguel-Tobal, Casado, Cano-Vindel y Spielberger, 1997).

Andreu, Peña y Graña (2002) realizaron un estudio psicométrico del Cuestionario de Agresión con una población española, los investigadores estimaron el grado de fiabilidad por consistencia interna u homogeneidad en su medida, siendo la escala de agresión física la que presentó una mayor precisión, alcanzando un coeficiente alpha de 0,86 .El resto de las escalas presentaron índices de consistencia interna 
menos elevados, en el caso de la escala de la ira presentó un coeficiente alpha de 0,77 , la escala de agresión verbal obtuvo 0,68 y la de hostilidad de 0,72. En relación a la validez de constructo presentada el análisis factorial confirmatorio, realizado a través de un modelo tetradimensional de ecuaciones estructurales, confirmó que, en términos generales, este cuestionario permite medir de forma válida la agresión física y verbal, la ira y la hostilidad en sujetos españoles.Aunque, tres de sus ítems deben ser re-elaborados en esta muestra dada su baja correspondencia con el modelo planteado, se puede afirmar que, por el momento, es un instrumento con suficiente y contrastada validez de constructo.

El objetivo de este estudio consistió en adaptar la versión española del Cuestionario de Agresión a la realidad peruana. Teniendo presente los problemas de violencia, conflicto y agresión que se observan en las escuelas, se establece el interés social, del presente estudio. Por tal motivo, a partir de la versión española del Cuestionario de Agresión, se aplicó a una muestra de estudiantes de distintas instituciones educativas de la costa, sierra y selva del Perú, con el fin de establecer la confiabilidad (consistencia interna) y validez de constructo (análisis factorial), además de obtener las normas y baremos (percentiles).

\section{Hipótesis general}

La versión española del Cuestionario de Agresión adaptada a la realidad peruana presenta confiabilidad y validez.

\section{Hipótesis específicas.}

1. La versión española del Cuestionario de Agresión adaptada a la realidad peruana presenta confiabilidad por consistencia interna.

2. La versión española del Cuestionario de Agresión adaptada a la realidad peruana presenta validez de constructo mediante el análisis factorial.

\section{MÉTODO}

Sujetos. La muestra del estudio estuvo compuesta por 3,632 adolescentes cuyas edades fluctuaban entre 10 y 19 años que asisten a instituciones educativas públicas de la costa, sierra y selva del Perú. El 47,9\% varones (1738 sujetos) y $52,1 \%$ mujeres (1894 sujetos). Con relación al nivel educativo de los sujetos, el $19,7 \%$ (717 sujetos) eran de $1^{\circ}$ de secundaria, el 20,0\% (725 sujetos) eran de $2^{\circ}$ de secundaria, el 20,4\% (742 sujetos) eran de $3^{\circ}$ de secundaria, el 20,0\% (725 sujetos) eran de $4^{\circ}$ de secundaria, y el $19,9 \%$ (721 sujetos) eran de $5^{\circ}$ de secundaria, las instituciones educativas seleccionados pertenecen a los departamentos de Amazonas, Ayacucho, Cuzco, Huancavelica, Huánuco, Junín,La Libertad, Lambayeque, Lima,Piura, Pucallpa, San Martín y Tacna.Dada la naturaleza del estudio se desarrolló un muestreo probabilístico bi-etápico por conglomerados (Pérez, 2000).La participación de los estudiantes fue anónima. 


\section{RESULTADOS}

A continuación se presentan los resultados hallados:

Tabla 1. Estadísticos de tendencia central

\begin{tabular}{lccccc}
\hline & $\begin{array}{c}\text { Agresividad } \\
\text { Física }\end{array}$ & $\begin{array}{c}\text { Agresividad } \\
\text { Verbal }\end{array}$ & Ira & Hostilidad & $\begin{array}{c}\text { Agresión (Esca- } \\
\text { la Total) }\end{array}$ \\
\hline $\mathrm{N}$ & 3632 & 3632 & 3632 & 3632 & 3632 \\
Media & 20,54 & 12,03 & 19,44 & 22,92 & 74,93 \\
Mediana & 20,00 & 12,00 & 19,00 & 23,00 & 75,00 \\
Moda & 17 & 13 & 19 & 24 & 79 \\
Desv. típ. & 6,258 & 3,711 & 4,726 & 5,888 & 16,096 \\
Varianza & 39,166 & 13,773 & 22,336 & 34,666 & 259,084 \\
\hline
\end{tabular}

Los resultados descriptivos que se observan en la Tabla 1, señalan que los sujetos de la muestra obtienen una media $=20,54$ y desvtip $=6,258$ en agresividad física; media $=12,03$ y desvtip $=3,711$, en agresividad verbal; media $=19,44$ y desvtip $=4,728$ en ira y media $=22,92$ y desvtip $=5,888$ en hostilidad. A nivel de la escala total se obtuvo media $=74,93$ y desvtip $=16,096$.

Tabla 2. Varianza total explicada

\begin{tabular}{cccc}
\hline \multirow{2}{*}{ Componentes } & \multicolumn{4}{c}{ Autovalores iniciales } \\
\cline { 2 - 4 } & Total & \% de la varianza & $\%$ acumulado \\
\hline 1 & 2,433 & 60,819 & 60,819 \\
\hline 2 &, 629 & 15,735 & 76,554 \\
\hline 3 &, 496 & 12,388 & 88,942 \\
\hline 4 &, 442 & 11,058 & 100,000 \\
\hline
\end{tabular}

Método de extracción: Análisis de Componentes principales.

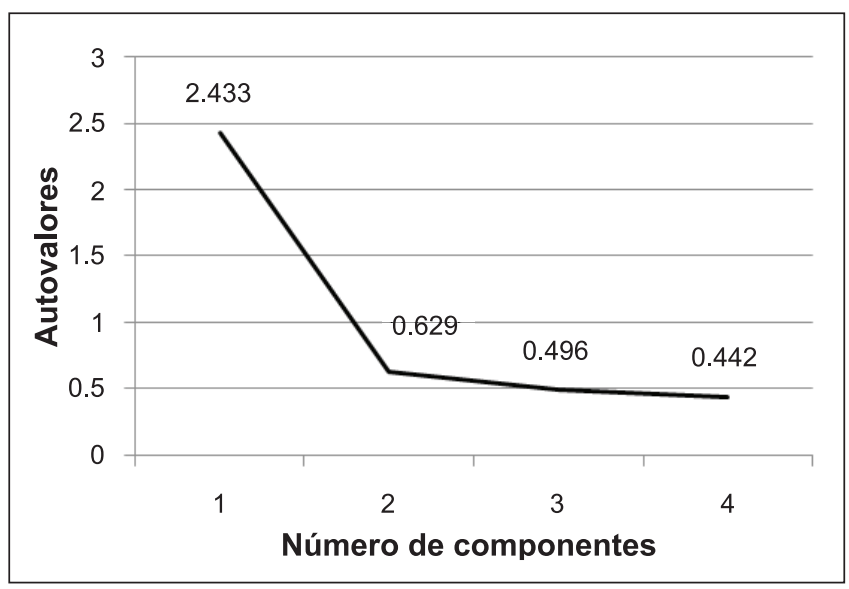

Figura 1. Gráfico de sedimentación 
El Análisis Factorial exploratorio que permita verificar la estructura de los principales factores (componentes) utilizando el método de los componentes principales se puede ver inicialmente a partir del gráfico de sedimentación (Figura 1). En la figura se indica que se debe extraer un componente principal que cumplen con el requisito señalado de valor propio (Autovalores) mayor que uno. Además como se observa en la Tabla 2, el análisis factorial arrojó un factor que llega a explicar el $60,819 \%$ de la varianza total acumulada, que demuestra una estructura del test compuesta por un factor que agrupa a cuatro componentes, resultado acorde al modelo propuesto por Arnold Buss.

Tabla 3. Matriz de componentes

\begin{tabular}{cc}
\hline \multirow{2}{*}{ Variables } & Componentes \\
\cline { 2 - 2 } & 1 \\
\hline Agresión Física &, 773 \\
\hline Agresión Verbal &, 770 \\
\hline Ira &, 812 \\
\hline Hostilidad &, 764
\end{tabular}

Método de extracción: Análisis de componentes principales.

1 componente extraído

En la Tabla 3, se presenta la matriz de componentes o de cargas factoriales que contiene la carga de los factores, es decir, la correlación existente entre cada variable y dicho factor. Las cargas indican el grado de correspondencia entre lavariable y el Factor (Agresión), es decir, que cargas altas indican quedicha variable es representativa para dicho factor. Se observa que para el caso de la variable Ira se le puede atribuir la carga factorial de 0,81 , siendo la más alta. Siendo lo aceptable que en el caso de las cargas factoriales, es que cada variable "cargará" más de 0,5 y cercano a 1 , sin embargo, valores como 0,4 se considera razonable. De este modo se observó que la carga factoriales es 0,773 en el caso de agresión física, 0,770 en agresión verbal y 0,764 en hostilidad.

Después de haber observado que los subtests se agrupan en torno a un factor subyacente al Cuestionario de Agresión, se pasó a determinar los índices de fiabilidad de la escala total y de las sub escalas del cuestionario.

Tabla 4. Estadísticos de fiabilidad

\begin{tabular}{ccc}
\hline & Alfa de Cronbach & N de elementos \\
\hline Escala total &, 836 & 29 \\
Subescala Agresión Física &, 683 & 9 \\
Subescala Agresión Verbal &, 565 & 5 \\
Subescala Ira &, 552 & 7 \\
Subescala Hostilidad &, 650 & 8 \\
\hline
\end{tabular}


La Tabla 4 presenta los coeficientes de fiabilidad, obtenidos en base al Coeficiente alphade Cronbach. Se observa un coeficientes de fiabilidad elevados para escala total $(\alpha=0,836)$, pero en el caso de la subescala agresión física $(\alpha=0,683)$, subescala agresión verbal $(\alpha=0,565)$, subescala Ira $(\alpha=0,552)$ y Hostilidad $(\alpha=$ $0,650)$.Los coeficientes de fiabilidad que se obtuvieron fueron diferentes a los obtenidos en la adaptación.

Tabla 5. Estadísticos total-elemento

\begin{tabular}{|c|c|c|c|c|}
\hline & $\begin{array}{l}\text { Media de la escala si } \\
\text { se elimina el ele- } \\
\text { mento }\end{array}$ & $\begin{array}{l}\text { Varianza de la escala } \\
\text { si se elimina el ele- } \\
\text { mento }\end{array}$ & $\begin{array}{l}\text { Correlación elemen- } \\
\text { to-total corregida }\end{array}$ & $\begin{array}{c}\text { Alfa de Cronbach si } \\
\text { se elimina el ele- } \\
\text { mento }\end{array}$ \\
\hline ítem 1 & 73,17 & 238,583 & ,379 & ,831 \\
\hline ítem 2 & 72,56 & 238,202 & ,352 &, 832 \\
\hline ítem 3 & 71,86 & 241,551 &, 242 &, 835 \\
\hline ítem 4 & 73,13 & 240,260 &, 324 & ,833 \\
\hline ítem 5 & 73,00 & 236,141 & ,396 &, 830 \\
\hline ítem 6 & 72,69 & 240,751 & ,307 & ,833 \\
\hline ítem 7 & 72,52 & 235,625 & ,406 &, 830 \\
\hline ítem 8 & 72,36 & 236,038 & ,380 &, 831 \\
\hline ítem 9 & 72,54 & 235,036 & ,406 &, 830 \\
\hline ítem 10 & 72,61 & 235,103 & ,435 & ,829 \\
\hline ítem 11 & 72,29 & 233,459 & ,431 & ,829 \\
\hline ítem 12 & 72,49 & 238,306 &, 344 &, 832 \\
\hline ítem 13 & 73,31 & 240,439 & ,354 & ,832 \\
\hline ítem 14 & 72,84 & 236,850 & ,388 & ,830 \\
\hline ítem 15 & 71,71 & 249,474 &, $072 *$ &, 840 \\
\hline ítem 16 & 71,89 & 236,908 & ,371 &, 831 \\
\hline ítem 17 & 72,47 & 237,155 &, 345 &, 832 \\
\hline ítem 18 & 73,23 & 238,690 & ,398 &, 830 \\
\hline ítem 19 & 73,04 & 238,119 & ,394 &, 830 \\
\hline ítem 20 & 72,42 & 235,046 & ,395 & 830 \\
\hline ítem 21 & 72,87 & 234,312 &, 436 &, 829 \\
\hline ítem 22 & 72,86 & 234,130 &, 452 & ,828 \\
\hline ítem 23 & 72,01 & 239,841 & ,258 & ,835 \\
\hline ítem 24 & 72,44 & 243,382 &, $179 *$ & ,838 \\
\hline ítem 25 & 72,56 & 235,988 & ,389 &, 830 \\
\hline ítem 26 & 72,31 & 233,915 & ,428 & ,829 \\
\hline ítem 27 & 73,24 & 237,841 & ,386 & ,831 \\
\hline ítem 28 & 71,86 & 239,577 & ,294 & ,834 \\
\hline ítem 29 & 73,05 & 236,736 & ,357 &, 831 \\
\hline
\end{tabular}

*correlación ítem-test $<0$. 
Como se observa en la Tabla 5, en la cual se presenta la correlación ítem-test corregida, siendo el criterio para aceptar o rechazar un ítem el que dicha correlación debe ser mayor o igual a 0,20 (Nunnaly y Bernstein, 1995). En la Tabla 4 las correlaciones ítem-test oscilan entre 0,072 y 0,452; observándose que el ítem 15 y el ítem 24 no obtienen correlaciones significativas con elpuntaje total de test, pero el aumento de la fiabilidad del test total no es mucho mayor si eliminamos dichos elementos así que se decide mantenerlo.

\section{DISCUSIÓN}

A partir de los resultados obtenidos, la fiabilidad observada en las cuatro subescalas (agresividad física, agresividad verbal, ira y hostilidad) que componen el Cuestionario de Agresión fueron satisfactorias. Para comprobar las hipótesis planteadas sobre la versión española del Cuestionario de Agresión adaptada a la realidad peruana presenta confiabilidad por consistencia interna y validez de constructo mediante el análisis factorial. Se observó que el grado de fiabilidad en relación con la precisión consistencia interna u homogeneidad en su medida, alcanzando un coeficiente de fiabilidad, obtenidos en base al Coeficiente alpha de Cronbach. Se observa un coeficiente de fiabilidad elevado para escala total $(\alpha=$ $0,836)$, pero en el caso de las subescalas son menores, es así que en agresión física $(\alpha=0,683)$, subescala agresión verbal $(\alpha=0,565)$, subescala ira $(\alpha=0,552)$ y Hostilidad $(\alpha=0,650)$.

De este modo se comprueba la hipótesis de fiabilidad debido a que puede considerarse suficiente para la evaluación del constructo Agresióny las variables asociadas al comportamiento agresivo en adolescentes. No obstante, se ha de tener en cuenta que la reformulación de algunos ítems (15 y 24) podría llevar a una mejoría en la fiabilidad de las subescalas de acuerdo con las correlaciones ítem test observadas.

Respecto a la validez de constructo presentada por el instrumento, mediante el análisis factorial exploratorio, que permite verificar la estructura de los principales factores utilizando el método de los componentes principales dio como resultado la extracción de un componente principal (agresión) que llega a explicar el 60,819\% de la varianza total acumulada, que demuestra una estructura del test compuesta por un factor que agrupa a cuatro componentes, resultado acorde al modelo propuesto por Arnold Buss, de este modo se comprueba la hipótesis de que la versión española del Cuestionario de Agresión adaptada a la realidad peruana presenta validez de constructo mediante el análisis factorial.

De acuerdo con estos resultados se puede concluir que el Cuestionario de Agresión permite medir el constructo agresión de acuerdo con la adaptación española realizada por Andreu, Peña y Graña (2002). Asimismo se ha podido demostrar que el instrumento en una muestra de estudiantes peruanos presenta características psicométricas con adecuada evidencia empírica que apoya la medición y sustento teóricos señalados por Buss y Perry (1992) para explorar los tipos de agresión (física y verbal), la hostilidad y la ira. 


\section{REFERENCIAS BIBLIOGRÁFICAS}

Andreu, J.;Peña, M. y Graña, J. (2002) Adaptación psicométrica de la versión española del Cuestionario de Agresión Psicothema, Vol. 14, $n^{o}$ 2, pp. 476-482.

Berkowitz, L. (1993). Aggression: Its causes, Consequences, and Control. New York: McGraw-Hill.

Buss A H. (1961) The psychology of aggression. New York: Wiley.

Buss A. y Perry, M. (1992)The aggression Questionnaire. Journal of Personality and Social Psychology. Vol. 63, $\mathrm{N}^{\mathrm{O}} 3,452-459$.

Bandura, A. (1976). Social learning analysis of aggression. En E. Ribes y A. Bandura (eds.): Analysis of delinquency and aggression. Hillsdale: Erlbaum.

Bandura A. (1977) Teoría del Aprendizaje Social. New Jersey, Englewood Cliffs, 1977.

Berkowitz, L. (1996). Agresión: causas, consecuencias y control. Bilbao: Desclée de Brouwer.

Diamond, E.L. (1982): The role of anger and hostility in essential hypertension and coronary heartdisease. Psychological Bulletin, 92, 410-433.

Fernández-Abascal, E.G. (1998): Psicología General. Motivación y Emoción. Madrid: Centro de Estudios Ramón Areces.

Izard, C.E. (1977): Human emotions. New York: Plenum Press.

Kaufmann, H. (1970). Agression and altruism.A psychological analisys. New York: Holt Rinebart and Winston Inc.

Nunnally, J. y Bernstein, I. (1995). Teoría psicométrica. México DF: MacGraw-Hill.

Miguel-Tobal, J.J., Casado, M.I., Cano-Vindel, A. y Spielberger, C.D. (1997): El estudio de la ira en los trastornos cardiovasculares mediante el empleo del Inventario de Expresión de Ira Estado-Rasgo. Ansiedad y Estrés, 3(1), 5-20.Peña, M. (2010) Conducta antisocial en adolescentes: factores de riesgo y de protección. Memoria para optar al grado de Doctor, Universidad Complutense de Madrid.

Pérez C. (2000) Técnicas de Muestreo Estadístico. México: Alfa Omega.

Plutchik, R.(1980): Emotion: A psychoevolutionary synthesis. New York: John Wiley.

Spielberger, C.D., Jacobs, G., Rusell, S.F. y Crane, R.S. (1983): Assessment of anger: The State-Trait Anger Scale. En J.N. Butcher y C.D. Spielberger (Eds.): Advances in Personality Assessment (vol. 2). Hillsdale, NJ: LEA. 
Anexo 1. Percentiles generales

\begin{tabular}{|c|c|c|c|c|c|c|c|c|c|}
\hline \multirow{2}{*}{\multicolumn{2}{|c|}{$\begin{array}{l}\text { ESCALA } \\
\text { TOTAL }\end{array}$}} & \multicolumn{8}{|c|}{ SUBESCALAS } \\
\hline & & \multicolumn{2}{|c|}{$\begin{array}{l}\text { AGRESIVIDAD } \\
\text { FÍSICA }\end{array}$} & \multicolumn{2}{|c|}{$\begin{array}{l}\text { AGRESIVIDAD } \\
\text { VERBAL }\end{array}$} & \multicolumn{2}{|c|}{ IRA } & \multicolumn{2}{|c|}{ HOSTILIDAD } \\
\hline PD & $\mathrm{Pc}$ & PD & $\mathrm{Pc}$ & PD & $\mathrm{PC}$ & PD & PC & PD & PC \\
\hline 112 & 99 & 38 & 99 & 21 & 99 & 31 & 99 & 36 & 99 \\
\hline 108 & 98 & 35 & 98 & 20 & 98 & 30 & 98 & 35 & 98 \\
\hline 105 & 97 & 34 & 97 & 19 & 96 & 29 & 97 & 34 & 97 \\
\hline 103 & 96 & 33 & 96 & 18 & 94 & 28 & 96 & 33 & 95 \\
\hline 102 & 95 & 32 & 95 & 17 & 90 & 27 & 94 & 32 & 94 \\
\hline 101 & 94 & 31 & 94 & 16 & 84 & 26 & 91 & 31 & 91 \\
\hline 99 & 93 & 30 & 92 & 15 & 78 & 25 & \begin{tabular}{|l|}
87 \\
\end{tabular} & 30 & 88 \\
\hline 98 & 92 & 29 & 89 & 14 & 70 & 24 & 83 & 29 & 84 \\
\hline 97 & \begin{tabular}{|l|}
91 \\
\end{tabular} & 28 & 87 & 13 & 60 & 23 & \begin{tabular}{|l|}
77 \\
\end{tabular} & 28 & 80 \\
\hline 96 & 90 & 27 & 83 & 12 & 50 & 22 & 71 & 27 & 74 \\
\hline 95 & \begin{tabular}{|l|}
89 \\
\end{tabular} & 26 & 80 & 11 & 40 & 21 & 63 & 26 & \begin{tabular}{|l}
69 \\
\end{tabular} \\
\hline 94 & \begin{tabular}{|l|}
88 \\
\end{tabular} & 25 & 76 & 10 & 31 & 20 & \begin{tabular}{|l|}
54 \\
\end{tabular} & 25 & 62 \\
\hline 93 & \begin{tabular}{|l}
87 \\
\end{tabular} & 24 & 71 & 9 & 22 & 19 & 45 & 24 & 56 \\
\hline 92 & 85 & 23 & 66 & 8 & 15 & 18 & \begin{tabular}{|l}
37 \\
\end{tabular} & 23 & 49 \\
\hline 91 & \begin{tabular}{|l|}
84 \\
\end{tabular} & 22 & 61 & \begin{tabular}{|l|}
7 \\
\end{tabular} & 9 & \begin{tabular}{|l}
17 \\
\end{tabular} & 29 & 22 & 42 \\
\hline 90 & \begin{tabular}{|l}
82 \\
\end{tabular} & 21 & 55 & 6 & 4 & 16 & 23 & 21 & 37 \\
\hline 89 & 81 & 20 & 49 & 5 & 1 & 15 & 17 & 20 & 31 \\
\hline 88 & \begin{tabular}{|l}
79 \\
\end{tabular} & 19 & 43 & & & 14 & 12 & 19 & 25 \\
\hline 87 & \begin{tabular}{|l|}
77 \\
\end{tabular} & 18 & 37 & & & 13 & 9 & 18 & 20 \\
\hline 86 & 75 & 17 & 31 & & & 12 & 6 & 17 & 16 \\
\hline 85 & 73 & 16 & 25 & & & 11 & 4 & 16 & 12 \\
\hline 84 & 71 & 15 & 20 & & & 10 & 2 & 15 & 9 \\
\hline 83 & 69 & 14 & 16 & & & 9 & 1 & 14 & 7 \\
\hline 82 & 66 & 13 & 11 & & & & & 13 & 5 \\
\hline 81 & 64 & 12 & 7 & & & & & 12 & 3 \\
\hline 80 & 61 & 11 & 4 & & & & & 11 & 2 \\
\hline 79 & 59 & 10 & 2 & & & & & 10 & 1 \\
\hline 78 & 56 & 9 & 1 & & & & & & \\
\hline 77 & 54 & & & & & & & & \\
\hline 76 & 51 & & & & & & & & \\
\hline 75 & 49 & & & & & & & & \\
\hline 74 & 47 & & & & & & & & \\
\hline 73 & 44 & & & & & & & & \\
\hline 72 & 42 & & & & & & & & \\
\hline 71 & 40 & & & & & & & & \\
\hline 70 & 37 & & & & & & & & \\
\hline 69 & 35 & & & & & & & & \\
\hline 68 & 32 & & & & & & & & \\
\hline 67 & 30 & & & & & & & & \\
\hline 66 & 28 & & & & & & & & \\
\hline 65 & 26 & & & & & & & & \\
\hline 64 & 25 & & & & & & & & \\
\hline
\end{tabular}




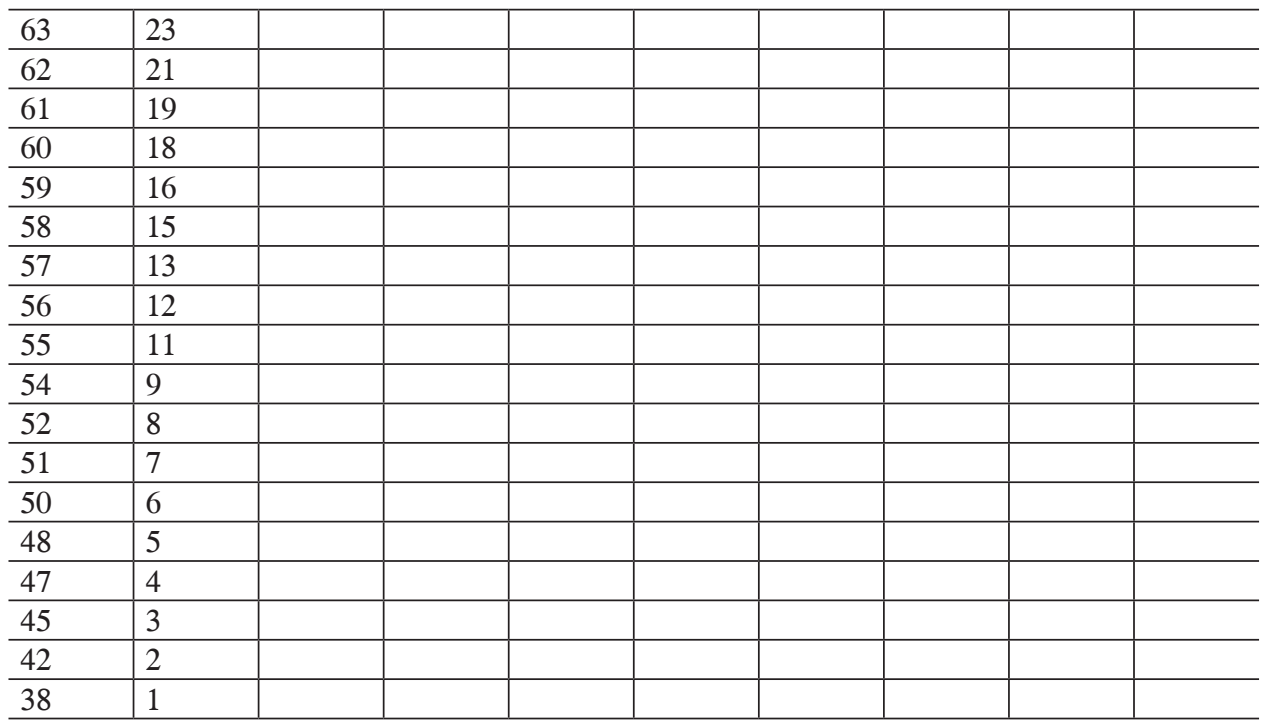

Anexo 2. Normas de ACUERdo a LA Distribución Z

\begin{tabular}{|c|c|c|c|c|c|}
\hline \multicolumn{2}{|c|}{$\begin{array}{l}\text { Límites de los niveles en la } \\
\text { distribución normal }\end{array}$} & \multirow[b]{2}{*}{ Nivel } & \multirow{2}{*}{$\begin{array}{l}\text { Cada nivel comprende } \\
\text { una desviación típica }(1 \mathrm{~s}) \\
\text { excepto los dos extremos }\end{array}$} & \multirow{2}{*}{\multicolumn{2}{|c|}{$\begin{array}{l}\text { Tanto por ciento aproxi- } \\
\text { mado de casos en la } \\
\text { Distribución Normal }\end{array}$}} \\
\hline $\mathrm{Z}$ & Percentil & & & & \\
\hline+1.50 & .933 & 5 & & \multicolumn{2}{|c|}{$7 \%$} \\
\hline \multirow[t]{2}{*}{+0.50} & \multirow[t]{2}{*}{.691} & 4 & 1desv. tip. & \multicolumn{2}{|c|}{$24 \%$} \\
\hline & & 3 & 1desv. tip & \multicolumn{2}{|c|}{$38 \%$} \\
\hline-0.50 & .308 & 2 & 1desv. tip & \multicolumn{2}{|c|}{$24 \%$} \\
\hline \multirow[t]{3}{*}{-1.50} & .066 & 1 & & \multicolumn{2}{|c|}{$7 \%$} \\
\hline & ESCALA & \multicolumn{2}{|c|}{ SUB ESCALAS } & \multirow[b]{2}{*}{ Ira } & \multirow[b]{2}{*}{ Hostilidad } \\
\hline & AGRESIÓN & $\begin{array}{l}\text { Agresividad } \\
\text { Física }\end{array}$ & $\begin{array}{l}\text { Agresividad } \\
\text { Verbal }\end{array}$ & & \\
\hline Muy Alto & 99 a Más & 30 a Más & 18 a Más & 27 a Más & 32 a Más \\
\hline Alto & $83-98$ & $24-29$ & $14-17$ & $22-26$ & $26-31$ \\
\hline Medio & $68-82$ & $16-23$ & $11-13$ & $18-21$ & $21-25$ \\
\hline Bajo & $52-67$ & $12-17$ & $7-10$ & $13-17$ & $15-20$ \\
\hline Muy Bajo & Menos a 51 & Menos a 11 & Menos a 6 & Menos a 12 & Menos a 14 \\
\hline
\end{tabular}


Anexo 3. Percentiles por Regiones

\begin{tabular}{|c|c|c|c|c|c|}
\hline \multicolumn{6}{|c|}{ ESCALA TOTAL } \\
\hline \multicolumn{2}{|c|}{ COSTA } & \multicolumn{2}{|c|}{ SIERRA } & \multicolumn{2}{|c|}{ SELVA } \\
\hline PD & PC & PD & $\mathrm{PC}$ & PD & $\mathrm{PC}$ \\
\hline 113 & 99 & 110 & 99 & 113 & 99 \\
\hline 108 & 98 & 106 & 98 & 109 & 98 \\
\hline 106 & 97 & 103 & 97 & 105 & 97 \\
\hline 104 & 96 & 102 & 96 & 104 & 96 \\
\hline 103 & 95 & 100 & 95 & 102 & 95 \\
\hline 102 & 94 & 99 & 94 & 101 & 94 \\
\hline 101 & 93 & 98 & 93 & 100 & 93 \\
\hline 100 & 92 & 97 & 93 & 99 & 93 \\
\hline 99 & 91 & 96 & 92 & 98 & 92 \\
\hline 98 & 90 & 95 & 91 & 97 & 91 \\
\hline 97 & 89 & 94 & 90 & 96 & 90 \\
\hline 96 & 88 & 93 & 89 & 95 & 88 \\
\hline 95 & 87 & 92 & 87 & 94 & 87 \\
\hline 94 & 86 & 91 & 85 & 93 & 86 \\
\hline 93 & 85 & 90 & 84 & 92 & 85 \\
\hline 92 & 84 & 89 & 82 & 91 & 83 \\
\hline 91 & 83 & 88 & 80 & 90 & 81 \\
\hline 90 & 82 & 87 & 78 & 89 & 79 \\
\hline 89 & 80 & 86 & 76 & 88 & 77 \\
\hline 88 & 78 & 85 & 74 & 87 & 75 \\
\hline 87 & 77 & 84 & 72 & 86 & 72 \\
\hline 86 & 75 & 83 & 70 & 85 & 70 \\
\hline 85 & 73 & 82 & 68 & 84 & 68 \\
\hline 84 & 71 & 81 & 65 & 83 & 66 \\
\hline 83 & 69 & 80 & 62 & 82 & 64 \\
\hline 82 & 66 & 79 & 59 & 81 & 62 \\
\hline 81 & 64 & 78 & 56 & 80 & 59 \\
\hline 80 & 62 & 77 & 54 & 79 & 57 \\
\hline 79 & 60 & 76 & 51 & 78 & 55 \\
\hline 78 & 57 & 75 & 49 & 77 & 52 \\
\hline 77 & 54 & 74 & 47 & 76 & 50 \\
\hline 76 & 52 & 73 & 45 & 75 & 48 \\
\hline 75 & 50 & 72 & 42 & 74 & 46 \\
\hline 74 & 48 & 71 & 40 & 73 & 43 \\
\hline 73 & 45 & 70 & 37 & 72 & 40 \\
\hline 72 & 42 & 69 & 34 & 71 & 38 \\
\hline 71 & 40 & 68 & 32 & 70 & 36 \\
\hline 70 & 38 & 67 & 30 & 69 & 33 \\
\hline 69 & 36 & 66 & 28 & 68 & 31 \\
\hline 68 & 33 & 65 & 26 & 67 & 28 \\
\hline 67 & 31 & 64 & 24 & 66 & 26 \\
\hline 66 & 30 & 63 & 22 & 65 & 25 \\
\hline 65 & 28 & 62 & 21 & 64 & 23 \\
\hline
\end{tabular}




\begin{tabular}{c|c|c|c|c|c}
\hline 64 & 26 & 61 & 19 & 63 & 21 \\
\hline 63 & 25 & 60 & 17 & 62 & 18 \\
\hline 62 & 23 & 59 & 16 & 61 & 16 \\
\hline 61 & 22 & 58 & 15 & 60 & 15 \\
\hline 60 & 20 & 57 & 14 & 59 & 13 \\
\hline 59 & 18 & 56 & 13 & 58 & 12 \\
\hline 58 & 16 & 55 & 11 & 57 & 10 \\
\hline 57 & 14 & 54 & 10 & 56 & 9 \\
\hline 56 & 13 & 53 & 9 & 55 & 8 \\
\hline 55 & 12 & 52 & 8 & 53 & 7 \\
\hline 54 & 10 & 51 & 7 & 52 & 6 \\
\hline 52 & 9 & 49 & 6 & 51 & 5 \\
\hline 51 & 8 & 47 & 5 & 50 & 4 \\
\hline 50 & 7 & 46 & 4 & 47 & 3 \\
\hline 49 & 6 & 44 & 3 & 44 & 2 \\
\hline 48 & 5 & 41 & 2 & 43 & 1 \\
\hline 46 & 4 & 40 & 1 & & \\
\hline 44 & 3 & & & & \\
\hline 42 & 2 & & & & \\
\hline
\end{tabular}

Anexo 4. Percentiles de subescalas por Regiones

\begin{tabular}{c|c|c|c|c|c|c|c|c|c|c|c}
\hline \multicolumn{7}{c|}{ AGRESIVIDAD FÍSICA } & \multicolumn{6}{c}{ AGRESIVIDAD VERBAL } \\
\hline \multicolumn{2}{c|}{ COSTA } & \multicolumn{2}{c|}{ SIERRA } & \multicolumn{2}{c|}{ SELVA } & \multicolumn{2}{c|}{ COSTA } & \multicolumn{2}{c}{ SIERRA } & \multicolumn{2}{c}{ SELVA } \\
\hline PD & PC & PD & PC & PD & PC & PD & PC & PD & PC & PD & PC \\
\hline 37 & 99 & 38 & 99 & 36 & 99 & 22 & 99 & 21 & 99 & 21 & 99 \\
\hline 35 & 98 & 36 & 98 & 35 & 98 & 21 & 98 & 20 & 98 & 20 & 97 \\
\hline 33 & 97 & 34 & 97 & 33 & 97 & 20 & 98 & 19 & 96 & 19 & 96 \\
\hline 32 & 96 & 33 & 96 & 32 & 96 & 19 & 96 & 18 & 94 & 18 & 93 \\
\hline 31 & 94 & 32 & 95 & 31 & 94 & 18 & 93 & 17 & 90 & 17 & 89 \\
\hline 30 & 91 & 31 & 94 & 30 & 92 & 17 & 89 & 16 & 85 & 16 & 83 \\
\hline 29 & 89 & 30 & 92 & 29 & 90 & 16 & 84 & 15 & 79 & 15 & 76 \\
\hline 28 & 86 & 29 & 90 & 28 & 88 & 15 & 77 & 14 & 71 & 14 & 68 \\
\hline 27 & 82 & 28 & 87 & 27 & 85 & 14 & 70 & 13 & 62 & 13 & 58 \\
\hline 26 & 78 & 27 & 84 & 26 & 81 & 13 & 60 & 12 & 51 & 12 & 47 \\
\hline 25 & 74 & 26 & 81 & 25 & 77 & 12 & 50 & 11 & 41 & 11 & 38 \\
\hline 24 & 69 & 25 & 77 & 24 & 73 & 11 & 41 & 10 & 31 & 10 & 29 \\
\hline 23 & 65 & 24 & 72 & 23 & 68 & 10 & 32 & 9 & 22 & 9 & 22 \\
\hline 22 & 61 & 23 & 66 & 22 & 62 & 9 & 23 & 8 & 14 & 8 & 14 \\
\hline 21 & 56 & 22 & 60 & 21 & 56 & 8 & 15 & 7 & 8 & 7 & 8 \\
\hline 20 & 50 & 21 & 54 & 20 & 51 & 7 & 9 & 6 & 4 & 6 & 4 \\
\hline 19 & 45 & 20 & 47 & 19 & 44 & 6 & 6 & 5 & 1 & 5 & 1 \\
\hline 18 & 39 & 19 & 41 & 18 & 37 & 5 & 2 & & & & \\
\hline 17 & 33 & 18 & 35 & 17 & 30 & & & & & & \\
\hline 16 & 27 & 17 & 29 & 16 & 24 & & & & & & \\
\hline 15 & 22 & 16 & 23 & 15 & 19 & & & & & & \\
\hline 14 & 18 & 15 & 18 & 14 & 14 & & & & & & \\
\hline 13 & 13 & 14 & 14 & 13 & 10 & & & & & & \\
\hline 12 & 8 & 13 & 10 & 12 & 7 & & & & & & \\
\hline 11 & 4 & 12 & 6 & 11 & 4 & & & & & & \\
\hline 10 & 2 & 11 & 3 & 10 & 2 & & & & & & \\
\hline 9 & 1 & 10 & 1 & 9 & 1 & & & & & & \\
\hline
\end{tabular}




\begin{tabular}{|c|c|c|c|c|c|c|c|c|c|c|c|}
\hline \multicolumn{6}{|c|}{ IRA } & \multicolumn{6}{|c|}{ HOSTILIDAD } \\
\hline COSTA & & SIERRA & & SELVA & & COSTA & & SIERRA & & SELVA & \\
\hline PD & $\mathrm{PC}$ & PD & $\mathrm{PC}$ & PD & $\mathrm{PC}$ & $\mathrm{PD}$ & $\mathrm{PC}$ & PD & $\mathrm{PC}$ & PD & $\mathrm{PC}$ \\
\hline 31 & 99 & 8 & 99 & 31 & 99 & 36 & 99 & 36 & 99 & 36 & 99 \\
\hline 30 & 98 & 9 & 98 & 30 & 98 & 35 & 98 & 35 & 98 & 35 & 98 \\
\hline 29 & 97 & 10 & 97 & 29 & 97 & 34 & 97 & 34 & 97 & 34 & 97 \\
\hline 28 & 96 & 11 & 95 & 28 & 96 & 33 & 95 & 33 & 96 & 33 & 95 \\
\hline 27 & 94 & 12 & 92 & 27 & 93 & 32 & 93 & 32 & 95 & 32 & 93 \\
\hline 26 & 91 & 13 & 89 & 26 & 89 & 31 & 90 & 31 & 92 & 31 & 91 \\
\hline 25 & 87 & 14 & 85 & 25 & 85 & 30 & 87 & 30 & 89 & 30 & 88 \\
\hline 24 & 82 & 15 & 80 & 24 & 81 & 29 & 84 & 29 & 85 & 29 & 84 \\
\hline 23 & 76 & 16 & 75 & 23 & 76 & 28 & 80 & 28 & 81 & 28 & 78 \\
\hline 22 & 70 & 17 & 69 & 22 & 69 & 27 & 75 & 27 & 75 & 27 & 72 \\
\hline 21 & 62 & 18 & 62 & 21 & 60 & 26 & 69 & 26 & 70 & 26 & 66 \\
\hline 20 & 54 & 19 & 53 & 20 & 51 & 25 & 63 & 25 & 63 & 25 & 60 \\
\hline 19 & 45 & 20 & 43 & 19 & 43 & 24 & 56 & 24 & 57 & 24 & 53 \\
\hline 18 & 37 & 21 & 35 & 18 & 35 & 23 & 49 & 23 & 50 & 23 & 47 \\
\hline 17 & 30 & 22 & 26 & 17 & 27 & 22 & 42 & 22 & 44 & 22 & 40 \\
\hline 16 & 24 & 23 & 20 & 16 & 20 & 21 & 37 & 21 & 39 & 21 & 33 \\
\hline 15 & 18 & 24 & 15 & 15 & 14 & 20 & 31 & 20 & 32 & 20 & 27 \\
\hline 14 & 13 & 25 & 10 & 14 & 9 & 19 & 26 & 19 & 26 & 19 & 21 \\
\hline 13 & 9 & 26 & 7 & 13 & 7 & 18 & 21 & 18 & 22 & 18 & 15 \\
\hline 12 & 6 & 27 & 4 & 12 & 4 & 17 & 17 & 17 & 18 & 17 & 11 \\
\hline 11 & 4 & 28 & 2 & 11 & 2 & 16 & 13 & 16 & 14 & 16 & 8 \\
\hline 10 & 2 & 29 & 1 & 10 & 1 & 15 & 9 & 15 & 10 & 15 & 6 \\
\hline \multirow[t]{6}{*}{9} & 1 & & & & & 14 & 7 & 14 & 7 & 14 & 4 \\
\hline & & & & & & 13 & 5 & 13 & 5 & 13 & 3 \\
\hline & & & & & & 12 & 3 & 12 & 4 & 12 & 2 \\
\hline & & & & & & 11 & 2 & 11 & 3 & 11 & 1 \\
\hline & & & & & & 10 & 1 & 10 & 2 & & \\
\hline & & & & & & & & 9 & 1 & & \\
\hline
\end{tabular}

\section{Cuestionario de Agresión (AQ)}

Nombres y Apellidos: Edad:

Sexo:

Institución Educativa: Grado de Instrucción:

\section{INSTRUCCIONES}

A continuación se presentan una serie de afirmaciones con respecto a situaciones que podrían ocurrirte. A las que deberás contestar escribiendo un aspa "X" $\mathbf{X}$ "según la alternativa que mejor describa tu opinión.

$\mathrm{CF}=$ Completamente falso para mí

$\mathrm{BF}=$ Bastante falso para mí

$\mathrm{VF}=\mathrm{Ni}$ verdadero, ni falso para mí

$\mathrm{BV}=$ Bastante verdadero para mí

$\mathrm{CV}=$ Completamente verdadero para mí 
Recuerdaque no hay respuestas buenas o malas,sólo interesa conocer laforma como tú percibes, sientes y actúas en esas situaciones.

\begin{tabular}{|c|c|c|c|c|c|}
\hline & $\mathrm{CF}$ & $\mathrm{BF}$ & $\mathrm{VF}$ & BV & $\mathrm{CV}$ \\
\hline \multicolumn{6}{|l|}{$\begin{array}{l}\text { 01. De vez en cuando no puedo controlar el impulso de golpear a otra } \\
\text { persona }\end{array}$} \\
\hline \multicolumn{6}{|l|}{$\begin{array}{l}\text { 02. Cuando no estoy de acuerdo con mis amigos, discuto abiertamente } \\
\text { con ellos }\end{array}$} \\
\hline \multicolumn{6}{|l|}{ 03. Me enojo rápidamente, pero se me pasa en seguida } \\
\hline \multicolumn{6}{|l|}{ 04. A veces soy bastante envidioso } \\
\hline \multicolumn{6}{|l|}{ 05. Si se me provoca lo suficiente, puedo golpear a otra persona } \\
\hline \multicolumn{6}{|l|}{ 06. A menudo no estoy de acuerdo con la gente } \\
\hline \multicolumn{6}{|l|}{ 07. Cuando estoy frustrado, muestro el enojo que tengo } \\
\hline \multicolumn{6}{|l|}{ 08. En ocasiones siento que la vida me ha tratado injustamente } \\
\hline \multicolumn{6}{|l|}{ 09. Si alguien me golpea, le respondo golpeándole también } \\
\hline \multicolumn{6}{|l|}{ 10. Cuando la gente me molesta, discuto con ellos } \\
\hline \multicolumn{6}{|l|}{$\begin{array}{l}\text { 11. Algunas veces me siento tan enojado como si estuviera a punto de } \\
\text { estallar }\end{array}$} \\
\hline \multicolumn{6}{|l|}{ 12. Parece que siempre son otros los que consiguen las oportunidades } \\
\hline \multicolumn{6}{|l|}{ 13. Suelo involucrarme en la peleas algo más de lo normal } \\
\hline \multicolumn{6}{|l|}{$\begin{array}{l}\text { 14. Cuando la gente no está de acuerdo conmigo, no puedo evitar } \\
\text { discutir con ellos }\end{array}$} \\
\hline \multicolumn{6}{|l|}{ 15. Soy una persona apacible } \\
\hline \multicolumn{6}{|l|}{$\begin{array}{l}\text { 16. Me pregunto por qué algunas veces me siento tan resentido por } \\
\text { algunas cosas }\end{array}$} \\
\hline \multicolumn{6}{|l|}{$\begin{array}{l}\text { 17. Si tengo que recurrir a la violencia para proteger mis derechos, lo } \\
\text { hago }\end{array}$} \\
\hline \multicolumn{6}{|l|}{ 18. Mis amigos dicen que discuto mucho } \\
\hline \multicolumn{6}{|l|}{ 19. Algunos de mis amigos piensan que soy una persona impulsiva } \\
\hline \multicolumn{6}{|l|}{ 20. Sé que mis «amigos» me critican a mis espaldas } \\
\hline \multicolumn{6}{|l|}{ 21. Hay gente que me provoca a tal punto que llegamos a pegarnos } \\
\hline \multicolumn{6}{|l|}{ 22. Algunas veces pierdo el control sin razón } \\
\hline \multicolumn{6}{|l|}{ 23. Desconfío de desconocidos demasiado amigables } \\
\hline \multicolumn{6}{|l|}{ 24. No encuentro ninguna buena razón para pegar a una persona } \\
\hline \multicolumn{6}{|l|}{ 25. Tengo dificultades para controlar mi genio } \\
\hline \multicolumn{6}{|l|}{$\begin{array}{l}\text { 26. Algunas veces siento que la gente se está riendo de mí a mis } \\
\text { espaldas }\end{array}$} \\
\hline \multicolumn{6}{|l|}{ 27. He amenazado a gente que conozco } \\
\hline \multicolumn{6}{|l|}{$\begin{array}{l}\text { 28. Cuando la gente se muestra especialmente amigable, me pregunto } \\
\text { qué querrán }\end{array}$} \\
\hline 29. He llegado a estar tan furioso que rompía cosas & & & & & \\
\hline
\end{tabular}


